\title{
Influence of beryllium carbide formation on deuterium retention and release
}

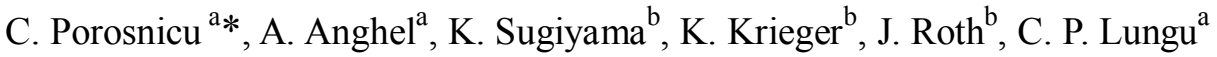 \\ ${ }^{a}$ National Institute for Laser, Plasma and Radiation Physics, Romania \\ ${ }^{b}$ Max-Planck-Institut für Plasmaphysik, Postfach 1533, D-8540 Garching, Germany
}

\begin{abstract}
The Inconel cladding tiles of the ITER-like-wall to be tested at JET will be coated by a beryllium layer. Carbon containing tiles will be also present. These materials are sputtered in the high flux $\left(10^{22} \mathrm{~m}^{-2} \mathrm{~s}^{-1}\right.$ or higher $)$ of the deuterium-tritium plasma. Ionized by the energetic electrons and with hydrogen isotope ions they will be implanted or re-deposited, creating composite layers.

In order to study the deuterium retention and release, mixed layers were prepared using the thermionic arc method.

Deuterium implantation was performed using a high current ion source at room temperature using a deuterium ion beam with energy of $200 \mathrm{eV} / \mathrm{D}$. Thermal Desorption Spectroscopy (TDS) analyses were correlated with the beryllium/carbon relative concentrations of the prepared films. At higher carbon concentrations the peak value from TDS spectra corresponding to beryllium was lower and an increased peak corresponding to the carbon was observed.
\end{abstract}

PSI-19 Keywords: Beryllium, Carbide, Co-deposition, Deuterium retention

JNM keywords: B0100, C0100, C0900, F0400, H0400, P0500

PACS. No. 52.40.Hf (Plasma-material interactions; boundary layer effects)

*Corresponding author:

Corneliu Porosnicu

National Institute for Laser, Plasma and Radiation Physics

Str, Atomistilor, No 409, PO Box MG-36,077125, Magurele, Romania

TEL: $+40-214574468$

FAX: $+40-214574468$

E-mail: corneliu.porosnicu@inflpr.ro

Presenting author:

Cristian Lungu

E-mail: cristian.lungu@inflpr.ro 


\section{Introduction}

Beryllium and carbon fiber composites (CFC) are candidate materials to be used on the ITER main chamber wall and divertor [1-2]. The tiles next to the divertor area will be subject to high fulx $\left(10^{22} \mathrm{~m}^{-2} \mathrm{~s}^{-1}\right.$ or higher $)$ of the deuterium-tritium plasma. Due to the sputtering and chemical erosion of these candidate materials, beryllium and hydrocarbon species particles can migrate into the plasma. They will be ionized by the energetic electrons and implanted or re-deposited on the divertor and on the shadowed areas together with hydrogen isotope ions.

Composite thin film depositions are used in order to obtain structures that have similar characteristics as the films deposited in different fusion reactor's areas. This type of films can be obtained under working conditions of the thermonuclear reactors, where parts of the walls are made of beryllium tiles or beryllium coated inconel tiles, and other reactor regions are made of carbon. The high energy plasma determines the migration and mixture of these elements. Beryllium carbide will easily be formed under reactor exposure conditions and therefore investigations on deuterium retention in Be-C systems are necessary.

This paper preparation provides a report on the preparation of Be-C films using the TVA deposition system. The relative Be-C concentration influence both on the retention and the desorption of the deuterium gas implanted into the prepared films using the high current ion source of IPP Garching at the energy of D ion beam of $600 \mathrm{eV} \mathrm{D}_{3}^{+}(200 \mathrm{eV} / \mathrm{D})$.

\section{Experimental}

The deposition method used is based on vacuum evaporation. This has the advantage of not having any buffer gas inside the deposition chamber, which makes the gas inclusions in the structure of the thin film to be practically absent. The method is called thermionic vacuum arc (TVA) [3]. The principle of this method is the ignition of the plasma in pure vapors of the 
material. The energy introduced in the system for the ignition of the plasma is given simultaneously by an electronic gun and a high voltage source. A Wehnelt cylinder focuses the electrons toward the anode crucible that has inside the material for deposition. The first vapors appear after a short period of time (20 - 30 seconds). A slight increase of the voltage, form $1 \mathrm{kV}$ to $1.5 \mathrm{kV}$, accelerates the electrons creating more vapors. Plasma appears in pure metal vapors when a specific value of the applied voltage; $1.5 \mathrm{kV}$ for beryllium and $2 \mathrm{kV}$ for carbon on the anode is reached. The ions created inside the plasma are accelerated toward the chamber walls and the substrates due to the potential difference between the plasma and the ground connected walls. The neutral atoms move outside the plasma due to the pressure gradient. The film is formed by both ions and neutral atoms.

During the entire coating process, a negative bias voltage was applied on the tiles, with a value of $-700 \mathrm{~V}$, which ensured a compact coating by rejecting the electrons and accelerating the positive ions from plasma [4].

In order to obtain films that have different $\mathrm{C}$-Be relative concentration, a new setup that uses two anodes at the same time was developed. The distance between the anodes was $20 \mathrm{~cm}$ and the sample holder-anodes distances were around $25 \mathrm{~cm}$, as the particle flux depends on the distance and the incident angle. The two anodes and the substrates were positioned so that a different particle flux was present for each sample in a deposition batch, as it is shown in figure 1. The samples were located collinearly above the two anodes at different distances toward the two evaporation sources ignited simultaneously. This way, we obtained C-Be films with almost continuum relative concentration variation in the same deposition batch.

Mirror polished fine grain graphite plates of dimensions $12 \mathrm{~mm}$ x $14 \mathrm{~mm}$ were used as substrates. In the first batch, the films were deposited at room temperature, while for the next an oven was used to heat the substrates at $725 \mathrm{~K}$. This way, the atoms and the ions from the plasma, reach the substrate without causing thermal shocks at the onset of the deposition due to the high 
mobility of the particles that arrive at the substrate. The lack of thermal shocks decreases the possibility of getting defects caused by mechanical stress [5].

Rutherford Backscattering Spectroscopy (RBS) and X-ray photoelectron spectroscopy (XPS) analyses were performed on the samples to asses the concentration of the two materials as well as the presence of oxygen and impurities and the films' composition and thickness.

For RBS measurements it had been used a ${ }^{4} \mathrm{He}$ energy beam of $2.6 \mathrm{MeV}$. The beam's energy value was chosen taking into account the interaction cross section of ${ }^{4} \mathrm{He}$ and beryllium to avoid the non-Rutherford interaction that may cause errors in the data interpretation. The fitting of the experimental data was done using SIMNRA code, developed at IPP Garching. [6]

The nuclear reaction $\mathrm{D}\left({ }^{3} \mathrm{He}, \mathrm{p}\right)^{4} \mathrm{He}$ offers information about the implanted deuterium inside the film. For the detection of the resulting particles ( $\alpha$ and protons), two detectors are beeing used, positioned at $102^{\circ}$ for protons and $135^{\circ}$ for $\alpha$ particles. The protons detector gives information about the total amount of deuterium inside the film, while the $\alpha$ particles detector is used to obtain the deuterium depth profile of the deuterium trapped inside the film. For these measurements, the energies used for the $3 \mathrm{He}$ beam were 690 and $800 \mathrm{keV}$. [6]

The XPS spectra were carried out by a Thermo Electron ESCALAB 250, USA using a monochromatized $\mathrm{Al} \mathrm{K \alpha}(1486.5 \mathrm{eV}) \mathrm{X}$-ray source, operated at a power of $150 \mathrm{~W}$. The survey spectra were performed with a pass energy of $70.0 \mathrm{eV}$ at a step of $1 \mathrm{eV}$, and high resolution scans were collected with a pass energy of $40.0 \mathrm{eV}$ at a step of $0.05 \mathrm{eV}$.

\section{Results and discussions}

\subsection{Elemental composition of the films}

The main aspect underlined by RBS measurements is the fact that samples with different C-Be ratios were obtained. Also, from the depth profiles obtained from RBS measurements it can be 
observed that for the room temperature substrates the film was oxidized only at the surface due to the air exposure after the deposition and at the interface, trapped inside before the deposition. For the heated substrates the oxygen present at the interface diffuses into the material, oxidizing the beryllium and the carbon in the whole film. (Figure 2).

XPS depth profiling spectra were recorded as function of time during sputtering using Ar ions with a density of $1 \mu \mathrm{A} / \mathrm{mm} 2$ at $3 \mathrm{kV}$ accelerating voltage, at normal angle incidence. XPS analysis was performed for one sample deposited at $725 \mathrm{~K}$ (Figure 3). Analysing the $105-120$ $\mathrm{eV}$ binding energy range, and assigning the Be1s peak at about $111 \mathrm{eV}$ to the metallic $\mathrm{Be}$ and the peak at about $113 \mathrm{eV}$ to the $\mathrm{Be}_{2} \mathrm{C}$ stable compound [7], the presence of the $\mathrm{Be}_{2} \mathrm{C}$ inside the whole film is being highlighted. Also, there can be observed oxidized beryllium (assigning the $115 \mathrm{eV}$ peak to the $\mathrm{BeO}$ compound), among the metallic beryllium and atomic carbon.

\subsection{Retention and desorption studies on Be-C films}

Due to the higher purity and low oxidation level, the samples deposited at room temperature were selected to be subjected to deuterium retention and desorption studies.

Deuterium implantation experiment was performed using the high current ion source of IPP Garching [8]. The energy of D ion beam was $600 \mathrm{eV} \mathrm{D}_{3}^{+}(200 \mathrm{eV} / \mathrm{D})$, and the incident direction was normal to the target surface. The implantation was carried out at a room temperature with the flux of $\sim 3 \times 10^{19} \mathrm{D} / \mathrm{m}^{2} \mathrm{~s}$. After certain continuum implantation $(2.09 \mathrm{x}$ $10^{21} \mathrm{D} / \mathrm{m}^{2}$ ), the amount of $\mathrm{D}$ retention was determined by nuclear reaction analysis (NRA). NRA analysis proved that the deuterium depth profile is highly influenced by the relative Be-C concentration. For high beryllium concentration, deuterium goes deeper inside the film (Figure $4(\mathrm{a}))$.

The total amount of deuterium retained (measured using the protons detector) as a function of the carbon concentration is shown in the Figure 5 and highly depends on the Be-C 
relative concentration. For high carbon concentration the amount of deuterium retained is lower. So, for the films that have $5 \%$ carbon composition the total amount of deuterium retained is $32 \%$ from the amount implanted, for the samples containing more than $50 \%$, the percentage of the deuterium amount retained is less than $10 \%$. It is presumed that this behavior occurred because of the carbon or carbide presence.

The samples corresponding to the two experimental points that do not follow the trend were analyzed before TDS and from the RBS depth profile it was observed that the films presented at the surface very high oxygen concentration (about $40 \%$ ) inside the whole film. It can be assumed that a $\mathrm{BeO}$ layer was formed modifying the retention mechanisms. How the samples accumulated oxygen is still unclear and although it is improbably, it is assumed that oxidation occurred during implantation process. These samples were not used for further studies.

Thermal desorption spectroscopy (TDS) experiment was performed for the implanted samples using the TESS facility at IPP Garching [9]. The experiment consists in heating the sample with $15 \mathrm{~K} / \mathrm{min}$ ramp, up to $1000 \mathrm{~K}$ followed by a 20 minutes hold. Then the sample was allowed to cool by itself. During the entire heating process, the amount of deuterium released from the film (as $\mathrm{D}_{2}$ and HD molecules) was measured.

TDS profiles for the Be-C samples showed clear dependence of deuterium release on the beryllium - carbon relative concentration. For implantation at room temperature and for high Be concentrations it was observed a pronounced $\mathrm{D}_{2}$ desorption peak between 425 and $475 \mathrm{~K}$, which corresponds to deuterium desorption from beryllium related trapping sites. At higher carbon/carbide concentrations this beryllium related desorption peak decreases and a correspondingly increasing peak between 925 and $975 \mathrm{~K}$ appears, which is attributed to retention of the carbon/carbide related trapping sites [10] (figure 6). 


\section{Summary}

Composite beryllium-carbon films were prepared using the thermionic vacuum arc technique, coating graphite substrates from two different sources, ignited simultaneously. Their characteristics are presumed to be similar with the future resulting inner wall surface after the thermo-nuclear plasma exposure inside ITER.

RBS measurements proved the fact that samples with different $\mathrm{C}$-Be ratios were obtained and that substrate temperature during deposition influences the oxygen concentration inside the films. The presence of the $\mathrm{Be}_{2} \mathrm{C}$ inside the whole film was highlighted by XPS analysis. Also, there can be observed oxidized beryllium.

Deuterium implantation was performed for the samples obtained at room temperature. Using Nuclear Reaction Analysis (NRA) was determined the retention of deuterium and deuterium depth profiles of the prepared samples.

The relative Be-C concentration influence both the retention and the desorption mechanisms. For low beryllium concentration into the mixed $\mathrm{Be}-\mathrm{C}$ layer the amount of deuterium was lower. The TDS spectra proved that the different samples different behavior was caused by different individual trapping sites for each of these two elements.

\section{Acknowledgements}

The authors appreciate I. Mustata, Th. Dürbeck, I. Jepu, C. Luculescu and V. Zaroschi for their scientific and technical assistance. This work is supported by EFDA task force under contract WP09-PWI-07-01/MEdC/B and WP10-PWI-06-02/MEdC. 


\section{References}

[1] R.Aymar, P. Babaraschi and Y. Shimomura, Plasma Phys. Control. Fusion 44 (2002) 519

[2] G. F. Matthews, P. Edwards, T. Hirai, et all., Phys. Scr. T128 (2007) 137

[3] C. P. Lungu, I. Mustata, V. Zaroschi et al., Phys. Scr. T128 (2007) 157

[4] Anghel, I. Mustata, C. Porosnicu, et al., C. P. Lungu, J. Nucl. Mater 385 ( 2009) 242-245

[5] C. P. Lungu, I. Mustata, V. Zaroschi, et all., Phys. Scr. T128 (March 2007) 157-161

[6] M. Mayer, SIMNRA User's Guide, Tech. Report IPP 9/113, Max-Plank-Institut fur Plasmaphysik, Garching, 1997

[7] P. Goldstraß and Ch. Linsmeier, Nucl. Instrum. Methods B 161-163 (2000) 411-414

[8] K. Krieger and J. Roth, J. Nucl. Mater 290-293 (2001) 107-111

[9] J. Roth, V.Kh. Alimov, A.V. Golubeva, et all., J. Nucl. Mater . 363-365 (2007) 822-826

[10]K. Sugiyama, J. Roth, A. Anghel et al., these proceedings 


\section{Figure captions}

\section{Figure 1:}

The schematic representation of the deposition system and the electrical connections used to obtain the Be-C composite films.

\section{Figure 2:}

Elemental depth profiles deduced from the RBS spectra of the deposited films at room temperature (a) and $725 \mathrm{~K}$ heated substrate (b).

\section{Figure 3:}

XPS depth profile of the Be-C composite film.

\section{Figure 4:}

Deuterium depth profiles for samples with different Be-C relative concentration (a) for high beryllium concentration, (b) for high carbon concentration.

\section{Figure 5:}

The total amount of deuterium retained as a function of the carbon concentration.

\section{Figure 6:}

$\mathrm{D}_{2}$ (a), HD (b) and total deuterium particles (c) Thermal desorption spectra for films with different beryllium - carbon relative concentrations, implanted at room temperature. 

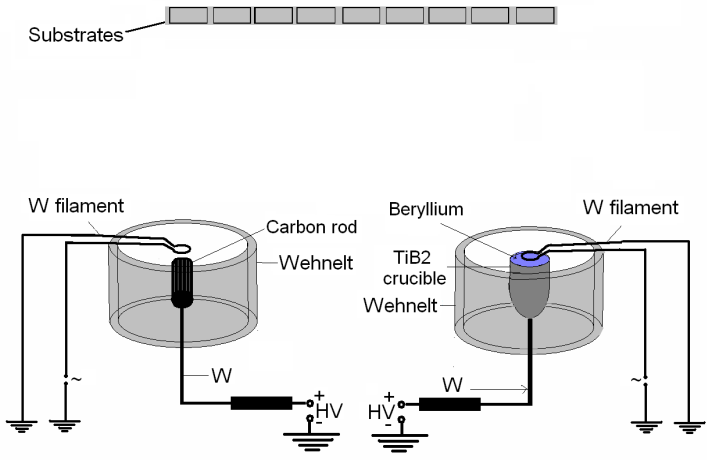

Fig. 1 The schematic representation of the deposition system and the electrical connections used to obtain the composite films

Fig. 1 Porosnicu et al. 

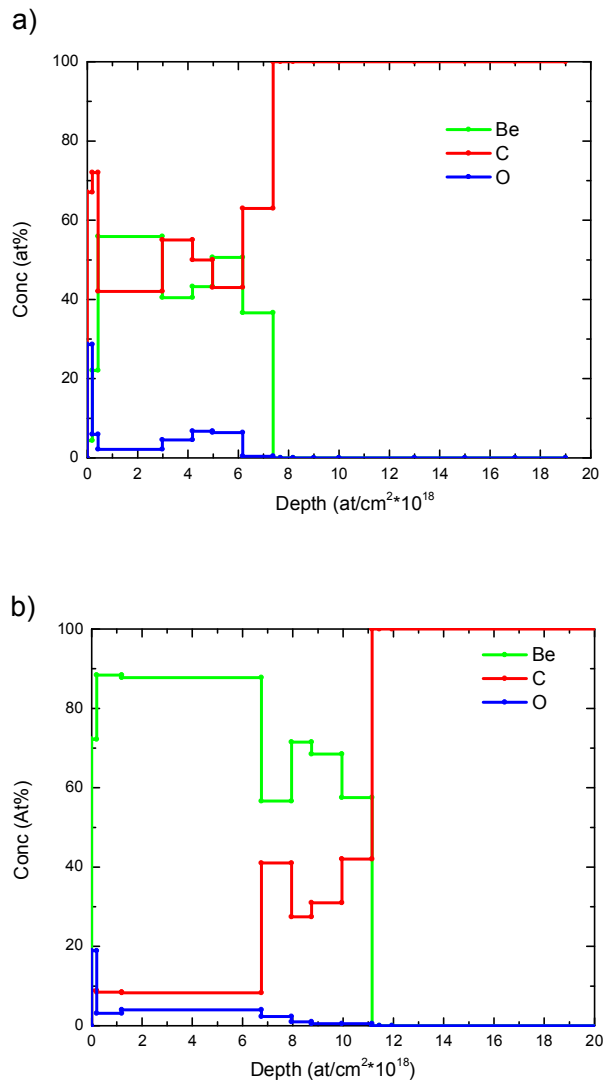

Fig. 2 Elemental depth profiles deduced from the RBS spectra for deposited films at room temperature (a) and at $725 \mathrm{~K}$ heated substrate (b).

Fig. 2 Porosnicu et al. 


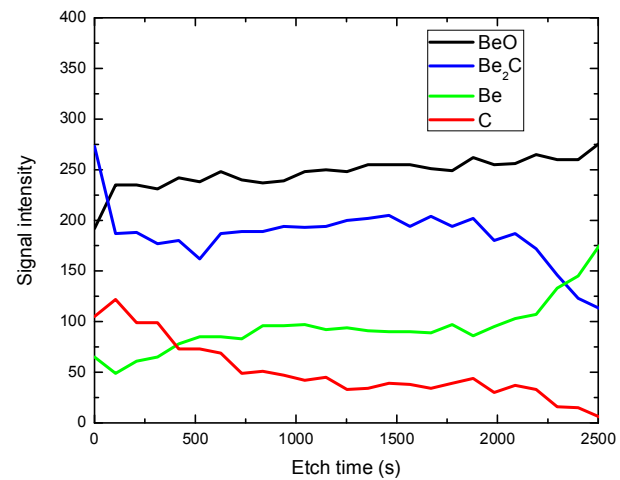

Fig. 3 XPS depth profile for Be-C composite film deposited at $725 \mathrm{~K}$

Fig. 3 Porosnicu et al. 

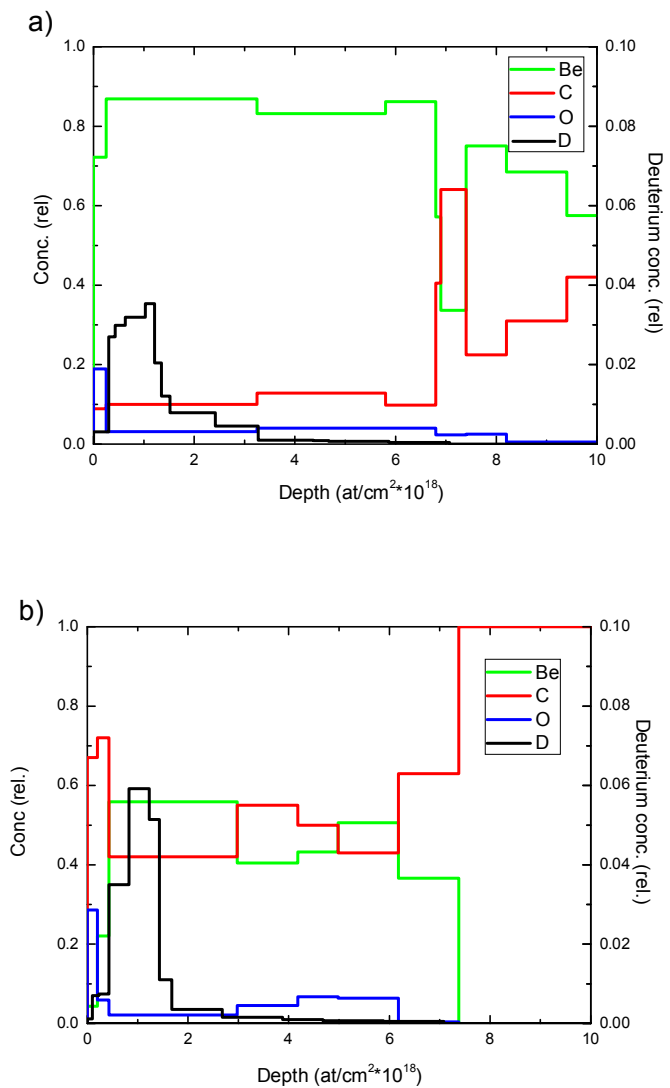

Fig. 4 Deuterium depth profiles for samples with different Be-C relative concentration for high beryllium concentration (a), and for high carbon concentration(b)

Fig. 4 Porosnicu et al. 


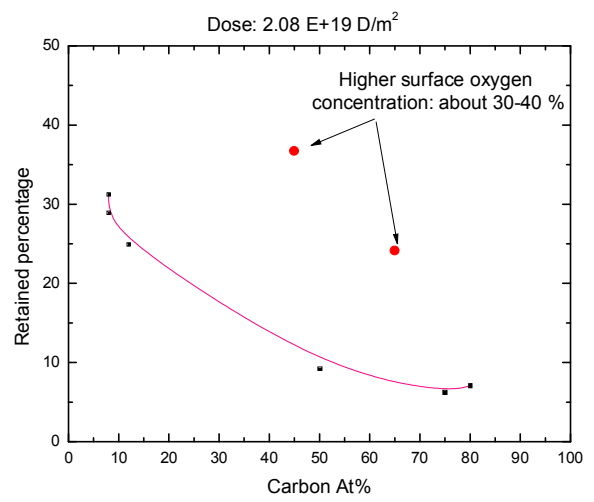

Fig. 5 The total amount of deuterium retained as a function of the carbon concentration

Fig. 5 Porosnicu et al. 

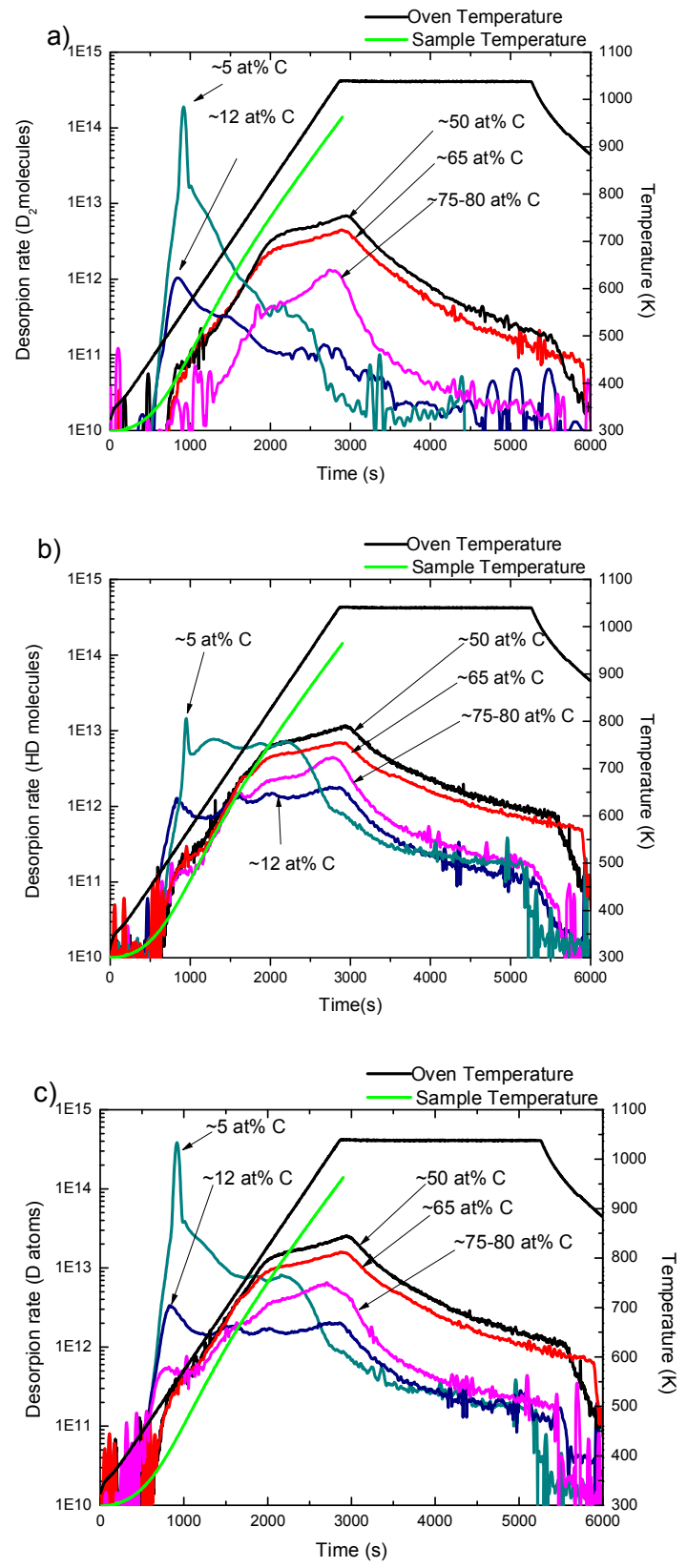

Fig. $6 \mathrm{D}_{2}$ (a), HD (b) and total deuterium particles (c) Thermal desorption spectra for films with different beryllium - carbon relative concentrations, implanted at room temperature.

Fig. 6 Porosnicu et al. 